\title{
A Single Mutation in the Acetylcholine Receptor $\delta$-Subunit Causes Distinct Effects in Two Types of Neuromuscular
} Synapses

\author{
Jee-Young Park, ${ }^{1}$ Meghan Mott, ${ }^{1}$ Tory Williams, ${ }^{1}$ Hiromi Ikeda, ${ }^{1}$ Hua Wen, ${ }^{2}$ Michael Linhoff,, 2 and Fumihito Ono ${ }^{1,3}$ \\ ${ }^{1}$ Section on Model Synaptic Systems, Laboratory of Molecular Physiology, National Institute on Alcohol Abuse and Alcoholism, National Institutes of \\ Health, Bethesda, Maryland 20892, ${ }^{2}$ Oregon Health and Science University, Portland, Oregon 97239, and ${ }^{3}$ Department of Physiology, Osaka Medical College, \\ Takatsuki, Japan, 569-0801
}

\begin{abstract}
Mutations in AChR subunits, expressed as pentamers in neuromuscular junctions (NMJs), cause various types of congenital myasthenic syndromes. In AChR pentamers, the adult $\varepsilon$ subunit gradually replaces the embryonic $\gamma$ subunit as the animal develops. Because of this switch in subunit composition, mutations in specific subunits result in synaptic phenotypes that change with developmental age. However, a mutation in any AChR subunit is considered to affect the NMJs of all muscle fibers equally. Here, we report a zebrafish mutant of the AChR $\delta$ subunit that exhibits two distinct NMJ phenotypes specific to two muscle fiber types: slow or fast. Homozygous fish harboring a point mutation in the $\delta$ subunit form functional AChRs in slow muscles, whereas receptors in fast muscles are nonfunctional. To test the hypothesis that different subunit compositions in slow and fast muscles underlie distinct phenotypes, we examined the presence of $\varepsilon / \gamma$ subunits in NMJs using specific antibodies. Both wild-type and mutant larvae lacked $\varepsilon / \gamma$ subunits in slow muscle synapses. These findings in zebrafish suggest that some mutations in human congenital myasthenic syndromes may affect slow and fast muscle fibers differently.
\end{abstract}

Key words: acetylcholine receptors; neuromuscular diseases; zebrafish

\section{Introduction}

Congenital myasthenic syndrome (CMS) is a neuromuscular disorder caused by mutations of multiple genes involved in the synapse formation/function in the neuromuscular junction (NMJ), including agrin (Huzé et al., 2009), ache (Ohno et al., 2001), rapsyn (Ohno et al., 2002), musk (Chevessier et al., 2004), and chrn (Engel et al., 2012). Symptoms of CMS include severe muscle weakness and fatigue. Transmission of signal at these synapses is performed by AChRs. AChRs are pentamers, composed of $\alpha 1_{2} \beta 1 \delta \gamma$ or $\alpha 1_{2} \beta 1 \delta \varepsilon$, depending on the developmental age. Embryonic $\gamma$ subunits are replaced by adult $\varepsilon$ subunits as the animal develops (Mishina et al., 1986; Walogorsky et al., 2012b). Causal mutations of myasthenic syndromes have been reported in all genes encoding AChR subunit (Engel et al., 2003). Mutations in the $\gamma$ subunit lead to phenotypes that change with development

Received Jan. 27, 2014; revised June 3, 2014; accepted June 18, 2014.

Author contributions: J.-Y.P. and F.O. designed research; J.-Y.P., M.M., T.W., H.I., and F.O. performed research; H.W. and M.L. contributed unpublished reagents/analytic tools; J.-Y.P. and F.O. analyzed data; J.-Y.P. and F.O. wrote the paper.

This work was supported by the intramural program at the National Institute on Alcohol Abuse and Alcoholism. We thank Drs. Alex Nechiporuk and Paul Brehm for kindly providing the love sofa mutant; and members of the Laboratory of Molecular Physiology for helpful discussions.

The authors declare no competing financial interests.

This article is freely available online through the J Neurosci Author Open Choice option.

Correspondence should be addressed to Dr. Fumihito Ono, MSC9411, National Institutes of Health/National Institute on Alcohol Abuse and Alcoholism, Bethesda, MD 20892-9411. E-mail: onof@mail.nih.gov.

DOI:10.1523/JNEUROSCI.0426-14.2014

Copyright $\odot 2014$ the authors $\quad 0270-6474 / 14 / 3410211-08 \$ 15.00 / 0$
(Robinson et al., 2013). In addition, we previously showed that a single mutation in the $\alpha 1$ subunit of zebrafish shows effects on the synaptic current that change with development. As the $\gamma$ subunit is replaced by $\varepsilon$, the synaptic phenotype is alleviated and the swimming of larvae recovers (Walogorsky et al., 2012a). However, mutations of AChR subunits are expected to affect synapses of both slow and fast muscle fibers equally.

Zebrafish have NMJs comparable with those of mammals. They are nicotinic, and postsynaptic AChRs are composed of subunits homologous to mammalian counterparts. Subunits corresponding to mammalian $\alpha 1, \beta 1, \gamma, \delta$, and $\varepsilon$ express and assemble in zebrafish. Some genes in zebrafish are duplicated because of the genome-wide duplication specific to teleosts (Meyer and Van de Peer, 2005). Among muscle-type AChR subunit genes in zebrafish, only $\beta 1$ has two duplicated copies, $\beta 1 \mathrm{a}$ and $\beta 1 \mathrm{~b}$. However, only $\beta 1 \mathrm{~b}$ is functional (Papke et al., 2012). Henceforth in this paper, we refer to $\alpha 1$ as $\alpha$ and $\beta 1 \mathrm{~b}$ as $\beta$ for simplicity.

The skeletal system in larval zebrafish trunks contains slow and fast muscle fibers: two muscle types that are also found in mammalian skeletal systems. Whereas slow and fast muscle fibers in mammals are intermingled and can be distinguished only by using histological techniques, such as ATPase staining (Brooke and Kaiser, 1970) or anti-myosin heavy chain antibody (Weiss et al., 1999), slow and fast muscle fibers of teleosts are spatially segregated and can easily be distinguished by their location and the orientation relative to the body axis (Bone, 1978; Luna and Brehm, 2006). The segregation and anatomical distinction of 
muscle fibers in zebrafish provide a unique opportunity to study the difference between these two types of muscle fibers, by analyzing the NMJs in preidentified types of muscle fibers.

Here, we isolated a novel mutant of the zebrafish AChR that expresses functional AChRs only in slow muscles. By analyzing this mutant, we showed that a single $\delta$ subunit mutation has different effects on AChRs of slow muscle and fast muscle fibers.

\section{Materials and Methods}

Fish lines. Zebrafish colonies were maintained following animal protocols at the National Institute on Alcohol Abuse and Alcoholism and the Oregon Health \& Science University. The love sofa mutant was generated by ENU mutagenesis by Dr. Alex Nechiporuk, Oregon Health and Science University, Portland, OR (Drerup and Nechiporuk, 2013) and was kindly provided. The allele sop $t j^{19 d}$ was used in the study (Granato et al., 1996). Mutants were crossed with the TAB strain (RRID:ZIRC_ZL1438) and maintained as male or female heterozygotes. Embryonic lethal homozygous larvae of sofa potato or love sofa were obtained by crossing male and female heterozygotes and used for experiments before their sex was determined.

Swimming analysis. High-speed image capturing of larval zebrafish escape response was performed with Photron camera at 1000 frames/s (Epley et al., 2008). Captured images were saved as JPEG files and processed with Photoshop (Adobe System).

Clones and expression. Constructs for expression of wild-type AChR $\delta$ subunit were previously described (Ono et al., 2004). It has a muscle-specific $\alpha$-actin promoter, and the expression was restricted to muscle cells. A point mutation for L332P was introduced to the clone with the QuikChange Lightening SiteDirected Mutagenesis Kit (Agilent Technologies). The construct was later confirmed to contain no other mutation than L332P by sequencing.

Electrophysiology. Miniature endplate current (mEPC) recordings from slow and fast muscles of zebrafish larvae were performed as previously described, with some modifications (Ono et al., 2001). The pipette solution contained Lucifer yellow (Sigma-Aldrich) at $1 \mathrm{mg} / \mathrm{ml}$. To minimize the movement of muscle fibers during the recording, an L-type calcium channel blocker, nifedipine (Sigma-Aldrich), was used instead of osmolality shock with formamide (Ono et al., 2001). It was shown recently that synaptic transmission of zebrafish NMJ depends on the P/Q type calcium channel (Wen et al., 2013), and $10 \mu \mathrm{M}$ nifedipine indeed did not inhibit mEPCs. Calcium channels in the excitation-contraction coupling were blocked by nifedipine, and stable recordings were possible. $100 \mathrm{~nm}$ TTX was added to the bath solution to block the spontaneous firing of motor neurons (Won et al., 2012). mEPC events were analyzed in MiniAnalysis (Synaptosoft). Events with slow rise and slow decay, which are reflections of synaptic events in neighboring cells, were excluded from analysis (Luna et al., 2004). For application of ACh, a glass electrode (opening $\sim 30 \mu \mathrm{m}$; filled with bath solution containing $30 \mu \mathrm{M}$ ACh and Lucifer yellow) was placed near the voltage-clamped muscle cell and positive pressure was applied by Picospritzer II (Parker Hannifin; 30 ms, 1 psi).

Immunohistochemistry. Immunohistochemistry methods, including information on antibodies, were previously described (Ikenaga et al.,
2011; Park et al., 2012). F59 antibody (Santa Cruz Biotechnology, catalog \#sc-32732 RRID:AB_670118) was used as a slow muscle marker, and nerve terminals were labeled with SV-2 antibody (Developmental Studies Hybridoma Bank, catalog \#sv2 RRID:AB_528480). For mAB35 staining, which recognizes an epitope in the $\mathrm{AChR} \alpha$ subunit, the incubation period for the primary antibody was $48 \mathrm{~h}$ (Sigma-Aldrich, catalog \#M217 RRID:AB_260473). A polyclonal antibody was raised against an epitope of NLISLNEKEETLTT, which is conserved between $\varepsilon$ and $\gamma$ subunits (GenScript). The specificity of the antibody was tested on the $\varepsilon$ knockout fish, which was generated using Transcription Activator-Like Effector Nuclease and will be reported elsewhere. The antibody staining was abolished at NMJs of the $\varepsilon$ knock-out fish (data not shown). $\alpha$-BTX staining was performed as described previously (Ono et al., 2001). All images were taken on the Zeiss 510 Meta Confocal microscope (Carl Zeiss Microimaging) with $40 \times$ C-Apo objective (NA 1.2) and analyzed in Photoshop (Adobe System).

\section{Results}

Through ENU mutagenesis, a new locomotion mutant was isolated. A complementation analysis with known locomotion mutants was performed, and the mutation failed to complement the sofa potato mutant, suggesting that the two mutants have a common affected gene. The sofa potato mutant has a missense muta- 
A

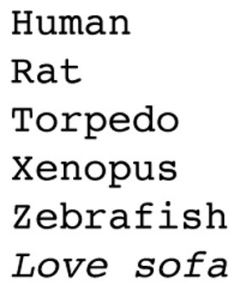

B
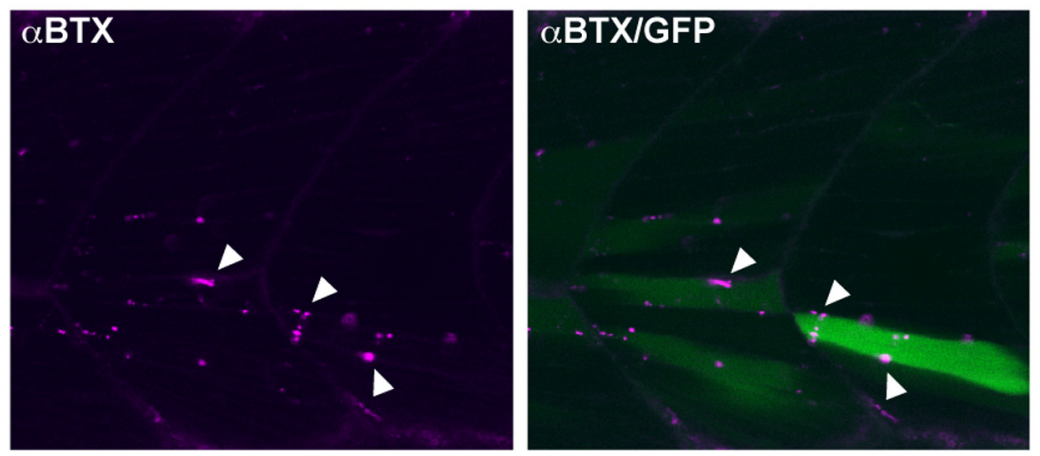

C
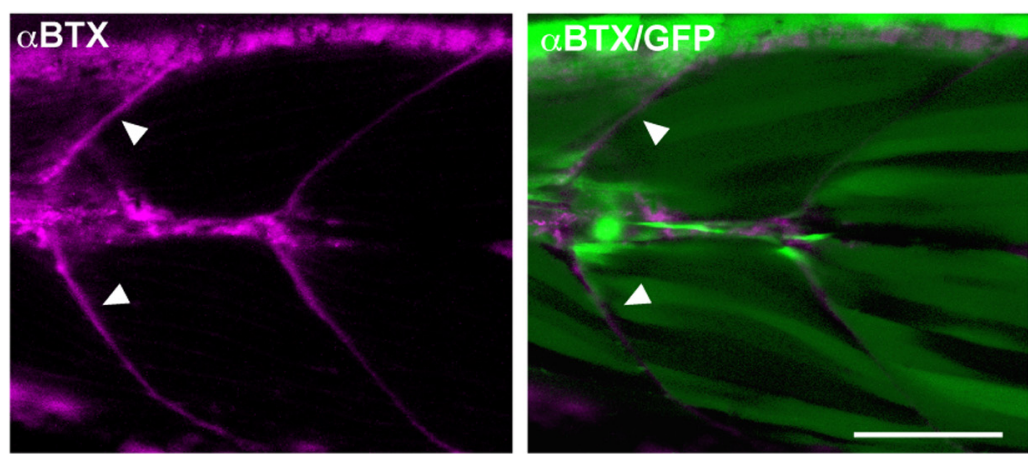

Figure 2. Genetic identification of the love sofa mutation. $\boldsymbol{A}$, Alignment of amino acid sequences of AChR $\delta$ subunit from human, rat, torpedo, Xenopus, wild-type zebrafish, and the love sofa mutant. The putative mutation of love sofa is located at the shadowed amino acid. In wild-type zebrafish, the amino acid is leucine, whereas in other species isoleucine or phenylalanine is found. In love sofa, it is changed to proline. The location of the L332P mutation in the $\delta$ subunit protein is shown in the right panel. $\boldsymbol{B}$, The wild-type $\delta$ subunit was expressed in mosaic fashion by injecting the gene construct into the love sofa mutant at one cell stage. Muscle fibers expressing the transgene, as marked by the cytoplasmic GFP (green), displayed distributed synapses (arrowheads) as marked by $\alpha$-BTX (magenta). C, When the $\delta$ subunit harboring the L332P mutation was expressed in the sofa potato mutant, $\alpha$-BTX staining visualized myoseptal synapses (arrowheads) but failed to exhibit distributed synapses. Magenta represents $\alpha$-BTX; green represents cytoplasmic GFP. Scale bar, $50 \mu \mathrm{m}$.

tion, L28P, in the AChR $\delta$ subunit (Ono et al., 2004). As a result of the L28P mutation, AChR pentamers do not form on the plasma membrane, and subunits are retained in the endoplasmic reticulum (Park et al., 2012). Because of the genetic commonality to sofa potato, we tentatively named the new mutant love sofa. Unlike the sofa potato mutant, which remains paralyzed until its death $\sim 6-8 \mathrm{~d}$ post fertilization ( $\mathrm{dpf}$ ), the love sofa mutant can move, although its tail beat and propulsion are weak (Fig. 1A).

When stained with $\alpha$-BTX, the love sofa mutant displayed a unique pattern of AChR distribution (Fig. 1B). In 3 dpf wild-type larvae, AChR clusters are observed at the edge of muscle cells and in the central regions of muscle cells as round, punctate spots (Fig. 1B). The former are called "myoseptal synapses" and the latter "distributed synapses" in previous studies (Lefebvre et al., 2007). The myoseptal synapses seem to be the only existent synapse in the love sofa mutant (arrowheads), and AChR clusterings at distributed synapses, in contrast, are almost completely absent. Therefore, synaptic transmission presumably occurs only at myoseptal synapses, which causes weak muscle contraction and compromised swimming. This pattern of $\alpha$-BTX staining is very different from that of sofa potato, which completely lacked AChRs on muscle cell surfaces (Ono et al., 2001).

We sequenced exons of the $\delta$ subunit gene in the love sofa mutant. We identified a putative missense mutation at L332, changing leucine to proline. The 332nd amino acid is either leucine (L), isoleucine (I), or phenylalanine (F) in the $\delta$ subunit of other species (Fig. 2A). The L332P mutation is located in the M3-M4 cytoplasmic loop, close to the M3 transmembrane region, and segregates strongly with the phenotype (Fig. 2A). It is therefore presumed causal to the phenotype.

To confirm that the $\delta$ subunit is the mutated gene, we performed a rescue experiment. Wild-type $\delta$ subunit was expressed in the love sofa mutant stochastically by injecting the DNA construct into fertilized eggs. After $3 \mathrm{~d}$ of incubation, some muscle cells in the developing larvae expressed wild-type $\delta$ subunits resulting from the transgene, as indicated by the cytoplasmic expression of GFP (green), and these cells formed distributed synapses (Fig. 2B, arrowheads). These data matched the complementation study and confirmed that the $\delta$ subunit gene is the responsible gene in the love sofa mutant.

To confirm that the L332P mutation is the causal mutation, we introduced a $\delta$ subunit harboring the L332P mutation $\left(\delta_{\mathrm{L} 332 \mathrm{P}}\right)$ into sofa potato mutants and examined whether we could phenocopy the love sofa mutant. Muscle cells without the $\delta_{\mathrm{L} 332 \mathrm{P}}$ transgene are genetically sofa potato, expressed no AChRs, and displayed no signal with $\alpha$-BTX staining (Ono et al., 2004). In contrast, muscle cells expressing the $\delta_{\mathrm{L} 332 \mathrm{P}}$ transgene, as indicated by the cytoplasmic GFP (green), showed $\alpha$-BTX-positive signals (magenta), but only along muscle cell edges (arrowheads), suggesting that these cells expressed only myoseptal synapses (Fig. $2 C$ ). Therefore, we propose the L332P mutation in the $\delta$ subunit is the causal mutation in love sofa.

Myoseptal synapses are found only in the superficial region of the trunk in zebrafish, whereas distributed synapses are found in deeper layers of muscle cells. This raises the possibility that myoseptal synapses are specific to slow muscles because slow muscle fibers form a single, superficial layer at $3 \mathrm{dpf}$ (Devoto et al., 1996). To test this possibility, we examined $\alpha$-BTX staining in wild-type larvae counterstained with the F59 antibody, which specifically labels slow muscle fibers (Fig. 3A) (Devoto et al., 1996; BrysonRichardson et al., 2005). In muscle cells positive for F59 signals, myoseptal signals of $\alpha$-BTX were observed (Fig. 3B). In deeper 
muscle cells negative for F59 signals, $\alpha$-BTX showed a pattern of distributed synapses (Fig. 3C). However, at the interface between slow muscles and fast muscles, distributed synapses were observed, which we could not assign to either of the two muscle fiber types. We therefore expressed wild-type $\delta$ subunit in the sofa potato mutant. The stochastic nature of the wild-type $\delta$ subunit expression allowed the observation of a single slow muscle fiber, whereas neighboring muscle cells were genetically sofa potato and completely lacked surface AChR expression. In slow muscle fibers expressing the wildtype $\delta$ subunit, only myoseptal synapses were observed (Fig. 3D). We conclude from these experiments that slow muscles have only myoseptal synapses and fast muscles have only distributed synapses. This suggests that, in the love sofa mutant, only slow muscles may have functional NMJs.

We recorded mEPCs from muscle fibers of the love sofa mutant using the whole-cell configuration patch clamp. The identification of fast or slow muscles was confirmed after patch-clamp recordings by the Lucifer yellow fluorescence contained in the pipette solution. Slow muscle fibers on the body surface are oriented parallel to the body axis, whereas deeper fast muscle fibers are oriented at an angle of $\sim 30^{\circ}$. In slow muscle cells, robust mEPCs were observed in both love sofa and wild-type larvae. Decay time constant (10\%-90\%) was fit to a single exponential, and the obtained time constant was plotted against the peak amplitude for each event. Representative plots from a single cell of love sofa and wild-type are shown in Figure $4 A$. When compared between groups of cells (Fig. 4B), the current amplitude was smaller (unpaired $t$ test; $p<0.01)$ in love sofa $(54.2 \pm 6.6 \mathrm{pA} ; n=$ 16 cells) compared with wild-type (108 \pm $19 \mathrm{pA} ; n=10$ cells), whereas the decay time constant was not different $(p=0.26)$ between love sofa $(4.25 \pm 0.51 \mathrm{~ms})$ and wild-type $(4.82 \pm 0.45 \mathrm{~ms})$. mEPCs recorded from fast muscles showed a striking difference between love sofa and wild-type. A majority of fast muscle cells in the love sofa mutant failed to exhibit any mEPCs $(n=13)$. In some fast muscles $(n=4)$, mEPCs with small amplitudes $(<60 \mathrm{pA})$ were observed. These events were highly infrequent, and statistical analysis from a single cell was difficult. When events from multiple fast cells were pooled, however, the smaller amplitude was evident compared with wild-type (Fig. 4C).

The lack of $\alpha$-BTX staining and the strong suppression of $\mathrm{mEPCs}$ in fast muscles raise three possibilities. First, terminals of motor neurons may not contact fast muscles or do not differentiate to release ACh. Second, pentamers may not assemble and express at synapses in fast muscles. Third, pentamers in fast muscles may assemble and reach the synapse, but not bind to $\alpha$-BTX

B

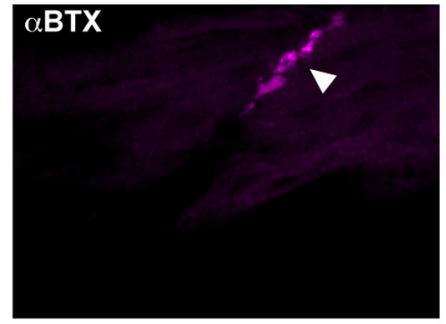

C
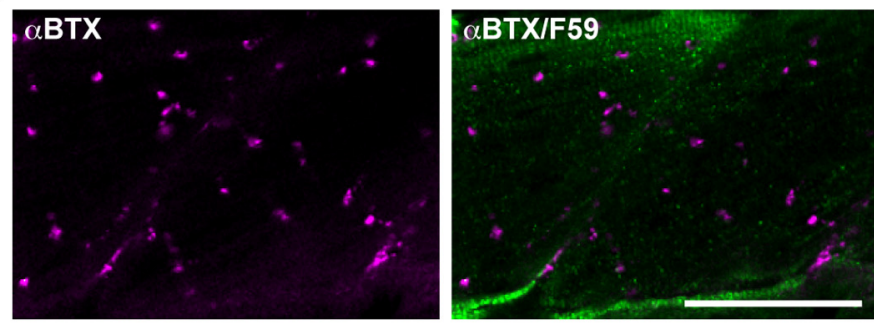

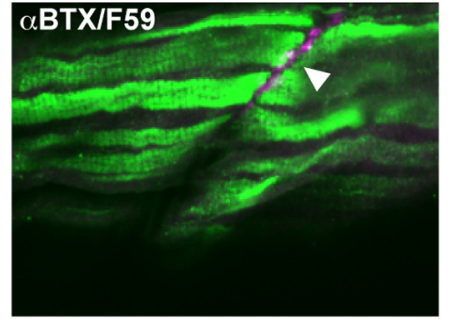

E

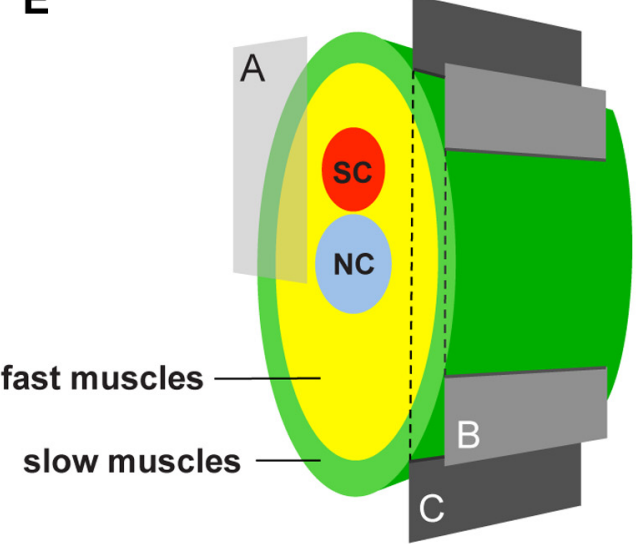

Figure 3. Different distribution of AChRs in slow and fast muscle fibers. $\boldsymbol{A}$, Cross section of a wild-type trunk stained with the F59 Optical slices of a wild-type larva near the surface $(\boldsymbol{B})$ or deeper $(\boldsymbol{C})$. Magenta represents $\alpha$-BTX staining; green represents the dignal. Scale bar, $50 \mu \mathrm{m}$. D, Stochastic expression of wild-type $\delta$ subunit in the sofa potato mutant. A slow must Because fast muscles in the neighboring layer lacked the transgene expression, only myoseptal $\alpha$-BTX staining was observed. Scale bar, $50 \mu \mathrm{m}$. $E$, Schematic cross section of the trunk of a larval zebrafish. Slow muscles are marked with green. Optical sections corresponding to $\mathrm{A}-\mathrm{C}$ are indicated with a box and planes. SC, Spinal cord; NC, notochord.

or ACh. To examine these possibilities, we performed immunohistochemistry, with antibodies reacting with a synaptic vesicle protein (SV2) or the AChR $\alpha 1$ subunit (mAb35) (Tzartos et al., 1981). Anti-SV2 antibody displayed signals at synapses of slow muscles and fast muscles, both in the love sofa mutant and the wild-type larvae. This indicates that nerve terminals of the love sofa mutant reached both slow and fast muscles and are differentiated. Staining with anti- $\alpha 1 \mathrm{AChR}$ in love sofa was observed in slow muscles (Fig. $5 A$, dashed box), which was expected from the $\alpha$-BTX staining. Interestingly, anti- $\alpha 1$ AChR staining (mAb35) exhibited signals also in fast muscles of the love sofa mutant (Fig. $5 A$, arrowheads) at locations colocalizing with nerve terminal endings (merged panel). This suggests that pentamers assemble and express at synapses in fast muscles of the love sofa mutant, but these pentamers cannot function, rendering the synapses almost silent.

To further confirm that the functional defect of fast muscles in the love sofa mutant is postsynaptic, we perfused slow and fast 
A

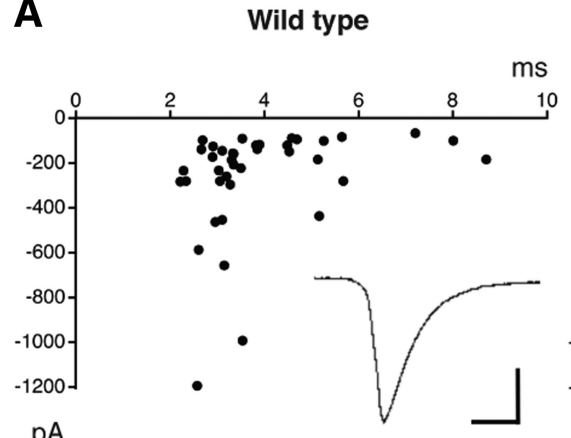

$\mathrm{pA}$

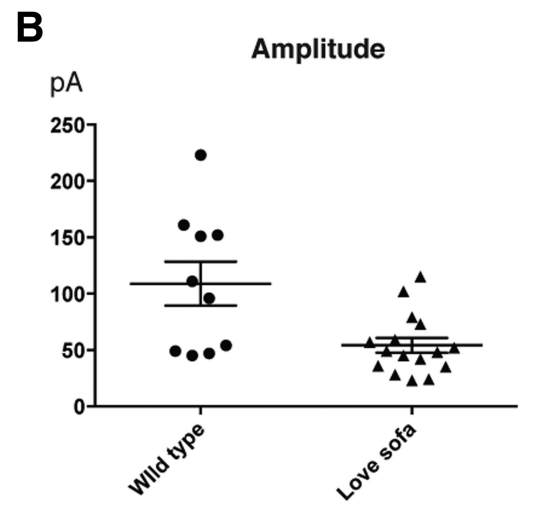

C Wild type

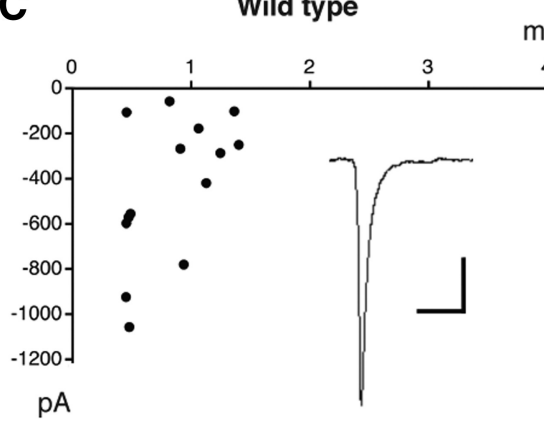

Love sofa

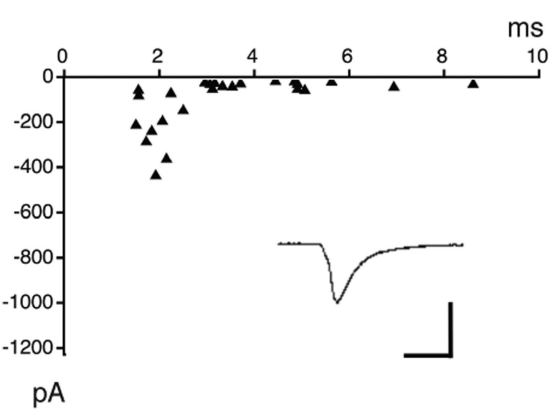

Decay time constant

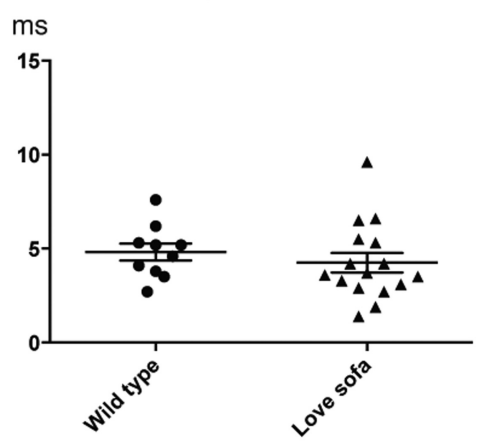

because of pentamer subunit compositions (i.e., $\alpha \beta \delta$ in slow fibers and $\alpha \beta \delta \varepsilon / \gamma$ in fast fibers). $\alpha \beta \delta_{\mathrm{L} 332 \mathrm{P}}$ in slow muscles are functional, whereas $\alpha \beta \delta_{\mathrm{L} 332 \mathrm{P}} \varepsilon / \gamma$ are nonfunctional.

To test this hypothesis, we generated an antibody against an epitope specific to the $\varepsilon$ and $\gamma$ subunit. In wild-type, fast muscles revealed the colocalization of the $\varepsilon / \gamma$ subunit and the $\alpha$-BTX staining (Fig. $6 A)$. In sharp contrast, slow muscles displayed the $\alpha$-BTX staining at muscle cell edges, but these locations did not have matching signals for the $\varepsilon / \gamma$ subunit. Likewise, fast muscles of love sofa showed positive clusters of the $\varepsilon / \gamma$ subunit, although $\alpha$-BTX staining is absent (Fig. 6B). Slow muscles in love sofa mutant again lacked $\varepsilon / \gamma$ subunits at synapses indicated by the $\alpha$-BTX staining (Fig. 6B). These results support our hypothesis that AChRs in slow muscles lacked the $\varepsilon / \gamma$ subunit in both wild-type and the love sofa mutant, and the presence of the $\varepsilon / \gamma$ subunit in the pentamer causes the specific effect of the $\delta_{\mathrm{L} 332 \mathrm{P}}$ mutation in fast muscles.

\section{Discussion}

We showed that a single mutation of the $\delta$ subunit, L332P, allows the synapse in slow muscles to function but renders those in fast muscles almost nonfunctional. Because of the distinct patterns of AChR distribution in slow and fast muscles of zebrafish (Fig. 3), which was first described in this paper, the love sofa mutant exhibits $\alpha$-BTX staining that is restricted to the myoseptal region (Fig. 1B). The characteristic pattern of $\alpha$-BTX staining restricted to the myoseptal region in love sofa larvae was observed as early as $1 \mathrm{dpf}$ (data not shown). Interestingly, the movement of the love sofa mutant at $1 \mathrm{dpf}$ was indistinguishable from that of wildtype, in contrast to the clearly defective swimming at $2 \mathrm{dpf}$ or later. This is in agreement with a previous study that showed that coiling, a type of movement specific to $1 \mathrm{dpf}$ embryos, depends on

muscles of the love sofa mutant under voltage clamp with a solution containing $30 \mu \mathrm{M} \mathrm{ACh}$. Slow muscles showed robust inward currents with the ACh application $(n=7)$. In contrast, fast muscles without mEPCs failed to display any inward current in response to the ACh application $(n=6)$. These data confirmed that the absence of mEPCs in the love sofa mutant results from the AChRs in fast muscle cells.

Why does a single $\delta$ subunit mutation have different effects on AChRs of slow muscles and fast muscles? We proposed previously that slow muscle fibers in zebrafish have subunit compositions lacking $\varepsilon$ or $\gamma$, based on the comparison of channel properties between native AChRs in zebrafish muscles and heterologously expressed AChRs in Xenopus oocytes (Mongeon et al., 2011). We therefore hypothesized that the $\delta_{\mathrm{L} 332 \mathrm{P}}$ subunit contained in both types of pentamers has distinct functional effects slow fibers (Naganawa and Hirata, 2011).

The identity of infrequent, small mEPCs in fast muscles of love sofa is unclear (Fig. 4C). Some of them may be reflections of mEPCs arising from neighboring slow muscle fibers transmitted through gap junctions (Luna et al., 2004). However, decays of mEPCs through gap junctions are usually prolonged, whereas some recorded mEPCs have decay time constants shorter than 2 ms, suggesting that the synaptic transmission occurred not in neighboring slow muscles but in the voltage-clamped fast muscle. In addition, we observed a very small number of distributed synapses in love sofa with $\alpha$-BTX staining (Fig. 1B). These cells may represent a special subpopulation of fast muscles, such as intermediate fibers. Muscle fibers with mixed metabolic characteristics of slow and fast fibers, called intermediate fibers, are reported 
in teleosts, including zebrafish adults (van Raamsdonk et al., 1982). Their distribution in embryos/larvae, however, remains unclear (Buss and Drapeau, 2000). In the stochastic rescue experiment (Fig. 3D), we also looked for a muscle fiber that exhibited a mixture of two types of AChR distribution, myoseptal and distributed. We did not find cells with a mixed distribution (data not shown). The identity of rare fibers that express distributed synapses in love sofa and their relationship to the infrequent $\mathrm{mEPCs}$ require further investigation.

An attractive hypothesis to explain the distinct effects of a single $\delta$ subunit mutation on two types of synapses is that the pentamer compositions are different. A parsimonious explanation is that the $\delta$ subunit is expressed in fast muscle fibers but not in slow muscle fibers. However, we reason that $\delta$ subunits are contained in synapses of both slow and fast muscle fibers. First, the $\delta_{\mathrm{L} 28 \mathrm{P}}$ mutation in the sofa potato mutant leads to the abolition of AChRs both in slow and fast muscle fibers (Ono et al., 2004). Second, the $\delta_{\mathrm{L} 322 \mathrm{P}} \mathrm{mu}-$ tation in the love sofa mutant had stronger effects in fast muscle fibers, but it also affected slow muscle fibers, reducing their current amplitude (Fig. 4B). Third, a combination of zebrafish AChR subunits lacking the $\delta$ subunit failed to form functional receptors when expressed heterologously in Xenopus oocytes (Mongeon et al., 2011). These data strongly suggest that pentamers in both fiber types contain the $\delta$ subunit. By using an antibody specific for $\varepsilon / \gamma$ subunits, we confirmed the previous study by showing that AChRs in slow muscles lack the $\varepsilon / \gamma$ subunit in wild-type. This difference of subunit composition was also observed in the love sofa mutant. These data support our hypothesis that different subunit compositions underlie distinct effects of the $\delta_{\mathrm{L} 332 \mathrm{P}}$ mutation in slow and fast muscles.

How can $\delta_{\text {L332P }}$ make $\alpha \beta \delta \varepsilon / \gamma$ pentamers nonfunctional while the function of $\alpha \beta \delta$ pentamers is retained? The $\delta_{\mathrm{L} 332 \mathrm{P}}$ subunit in the $\alpha \beta \delta_{\mathrm{L332} 2 \mathrm{P}} \varepsilon / \gamma$ pentamer may affect the AChR function (Fig. $6 C)$. In human congenital myasthenic syndromes, AChR mutations change the channel gating, the binding of ligand, or the expression of AChR to the membrane surface (Ohno et al., 1996; Wang et al., 2000). A combination of these effects can also be observed by a single mutation. The lack of binding to $\alpha$-BTX in love sofa fast muscles suggests that the binding of ligand is changed because the binding domains for ACh and $\alpha$-BTX are identical (Balass et al., 1997; Harel et al., 2001). The expression of $\mathrm{AChR}$, on the other hand, does not seem to be strongly affected because mAb35 staining revealed the existence of pentamers at synapses of fast muscles (Fig. $5 A$ ). In the $\alpha \beta \delta \varepsilon / \gamma$ composition, two $\alpha$ s and a single subunit of $\beta, \delta$, and $\varepsilon$ (or $\gamma$ ) make up the pentamer, and the binding with ACh as well as $\alpha$-BTX occurs with amino acid residues located between $\alpha \delta$ and $\alpha \varepsilon$ (Fig. 6C) (Blount and Merlie, 1989). The $\beta$ subunit is a structural subunit

Slow muscle
Wild type
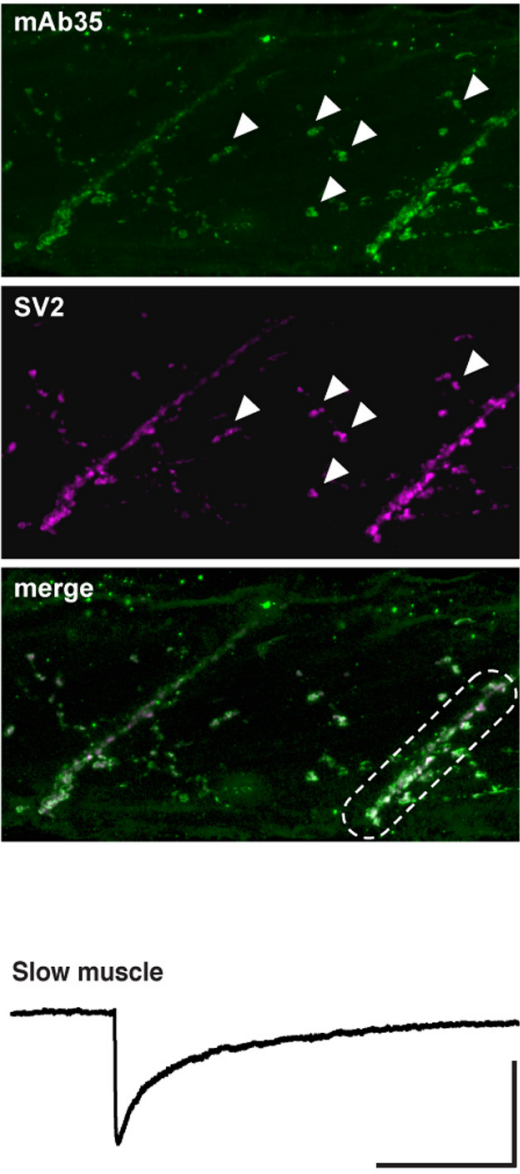

Fast muscle
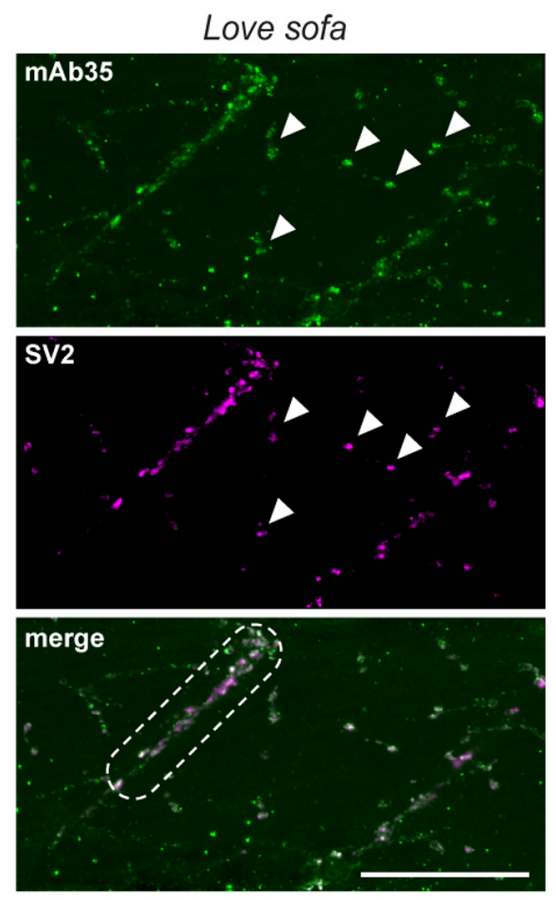

C

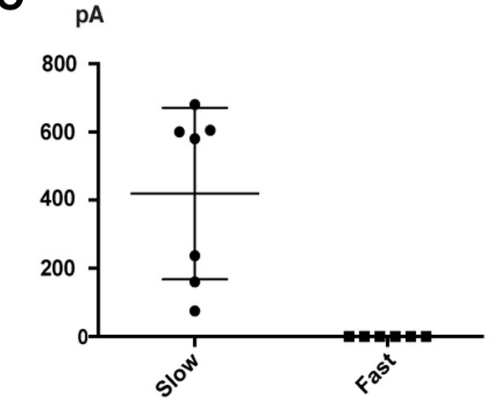

Figure 5. Nonfunctional AChRs in fast muscles of the love sofa mutant. $\boldsymbol{A}$, Wild-type (left) and love sofa (right) larvae were doubly labeled with mAb35 antibody (green) and SV2 antibody (magenta). Boxes of dashed lines indicate myoseptal synapses in clamped slow and fast muscles in response to the application of $30 \mu \mathrm{m} \mathrm{ACh}$. Calibration: $5 \mathrm{~s}, 500 \mathrm{pA}$. C, Amplitudes of ACh-induced currents in slow $(n=7)$ and fast muscles $(n=6)$ are shown. Each dot represents a muscle cell.

that does not contribute to ligand binding. The $\alpha \beta \delta$ composition, in contrast, likely contains two $\alpha$ s and two $\delta$ s (Fig. $6 C$ ) (Charnet et al., 1992). It is expected that slow muscles of love sofa pentamers contain two $\delta_{\mathrm{L} 332 \mathrm{P}} \mathrm{s}$, whereas fast muscles contain only one $\delta_{\mathrm{L} 332 \mathrm{P} \text {. }}$ It is therefore surprising and somewhat counterintuitive that the pentamer with two mutant $\delta$ s is functional, whereas that with one mutant $\delta$ is strongly affected. However, the location of the L332P mutation is not close to the ligand-binding domain, and the mutation will not only affect local areas surrounding the mutated amino acid. Instead, the effect of the mutation on the overall AChR structure will be the result of a global change. While the crystal structure of acetylcholine binding protein (AChBP) elucidated how extracellular components of AChRs assemble (Brejc et al., 2001), the contribution of the M3-M4 intracellular loop to the overall structure/function of pentamers remains less well understood. This region generally allows larger flexibility, making it a preferable site of insertion for fluorescent molecules (Nashmi et al., 2003; Epley et al., 2008). The L332 may be too close to the M3 to allow for such flexibility. There are also examples of M3-M4 loop mutations that lead to 
A
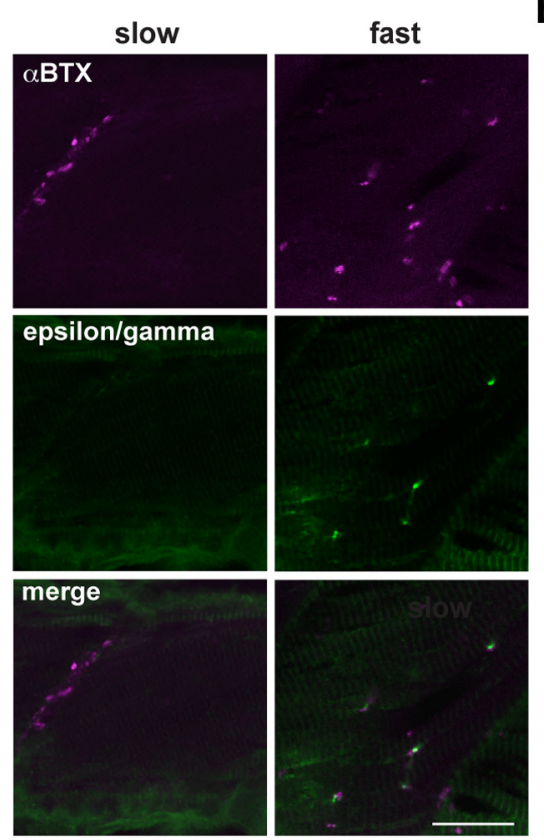

C

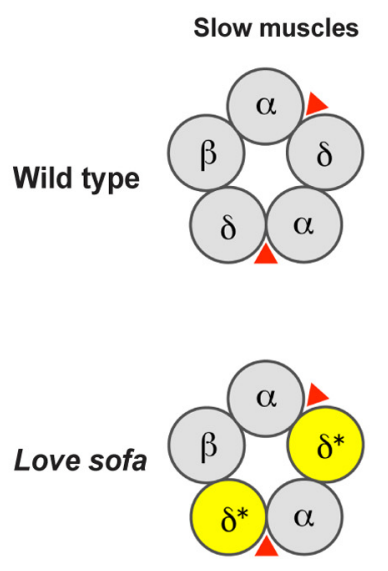

Figure 6. Distinct subunit compositions of AChRs in slow and fast muscles underlie the fiber-type specific phenotypes in the love sofa mutant. Wild-type $(\boldsymbol{A})$ and love sofa $(\boldsymbol{B})$ larvae were doubly labeled with an antibody for $\varepsilon / \gamma$ subunits (green) and $\alpha$-BTX (magenta). Optical sections corresponding to slow muscle fibers and fast muscle fibers are shown. Scale bar, $20 \mu \mathrm{m}$. $\boldsymbol{C}$, A schematic depicting the hypothesis. In wild-type larvae (top), pentamers of slow muscles comprise $\alpha \beta \delta$, whereas those in fast muscle comprise $\alpha \beta \delta \varepsilon / \gamma$. Red triangle represents the ligand (ACh or $\alpha$-BTX). In the love sofa mutant (bottom), the $\delta$ subunit harbors a L332P mutation (shown with an asterisk and yellow). As a result, the binding of ligand to the pockets between $\alpha$ and $\delta$ is inhibited in fast muscles.

low AChR expressions (Engel et al., 1999). Interestingly, a six residue in-frame duplication in the M3-M4 cytoplasmic loop close to M3 leads to a mode switching of channel kinetics in the human $\varepsilon$ subunit (Milone et al., 1998). The effect of L332P mutation on the ligand binding and channel kinetics, which is dependent on the subunit composition, may provide new information on the structure of pentamers.

Subunit compositions of AChRs in human slow muscles have not been studied separately from fast muscles; and to the best of our knowledge, a type of human myasthenic syndrome that predominantly affects either slow or fast muscles has not been reported. Interestingly, in $\varepsilon$ knock-out mice, the proportion of slow muscle fibers increases, suggesting a functional link between the receptor subunit composition and the fiber type (Jin et al., 2008).
A generally held belief was that the only two possible combinations of subunits in vertebrate NMJs were $\alpha \beta \delta \varepsilon$ and $\alpha \beta \delta \gamma$. However, the combination of $\alpha \beta \delta$ leads to a functional channel in heterologous expression systems (Charnet et al., 1992), and we recently proposed that this combination works in NMJs in vivo, which was further confirmed in the present study (Mongeon et al., 2008). It will be highly intriguing to examine whether $\alpha \beta \delta$ pentamers also express in (sub)populations of human slow muscle fibers. Moreover, some muscle fibers found in the mammalian extraocular muscle show special characteristics, including the lack of action potentials (Chiarandini and Stefani, 1979) or the location of NMJs at myotendinous junctions (Zimmermann et al., 2011). Molecular identity of AChRs in these extraocular fibers remains unknown. If they have AChR subunit compositions distinct from fast muscle fibers, patients with certain types of mutations may present symptoms caused by phenotypes specific to such fibers. We therefore propose that consideration of NMJs containing $\alpha \beta \delta$ pentamers may be beneficial to better understand congenital myasthenic syndromes.

\section{References}

Balass M, Katchalski-Katzir E, Fuchs S (1997) The alpha-bungarotoxin binding site on the nicotinic acetylcholine receptor: analysis using a phage-epitope library. Proc Natl Acad Sci U S A 94:6054-6058. CrossRef Medline

Blount P, Merlie JP (1989) Molecular basis of the two nonequivalent ligand binding sites of the muscle nicotinic acetylcholine receptor. Neuron 3:349-357. CrossRef Medline

Bone Q (1978) Locomotor muscle. In: Fish physiology Vol. II (Randall H, ed), pp 1-34. New York: Academic.

Brejc K, van Dijk WJ, Klaassen RV, Schuurmans M, van Der Oost J, Smit AB, Sixma TK (2001) Crystal structure of an ACh-binding protein reveals the ligand-binding domain of nicotinic receptors. Nature 411:269-276. CrossRef Medline

Brooke MH, Kaiser KK (1970) Three "myosin adenosine triphosphatase" systems: the nature of their $\mathrm{pH}$ lability and sulfhydryl dependence. J Histochem Cytochem 18:670-672. CrossRef Medline

Bryson-Richardson RJ, Daggett DF, Cortes F, Neyt C, Keenan DG, Currie PD (2005) Myosin heavy chain expression in zebrafish and slow muscle composition. Dev Dyn 233:1018-1022. CrossRef Medline

Buss RR, Drapeau P (2000) Physiological properties of zebrafish embryonic red and white muscle fibers during early development. J Neurophysiol 84:1545-1557. Medline

Charnet P, Labarca C, Lester HA (1992) Structure of the gamma-less nicotinic acetylcholine receptor: learning from omission. Mol Pharmacol 41: 708-717. Medline

Chevessier F, Faraut B, Ravel-Chapuis A, Richard P, Gaudon K, Bauché S, Prioleau C, Herbst R, Goillot E, Ioos C, Azulay JP, Attarian S, Leroy JP, Fournier E, Legay C, Schaeffer L, Koenig J, Fardeau M, Eymard B, Pouget J, et al. (2004) MUSK, a new target for mutations causing congenital myasthenic syndrome. Hum Mol Genet 13:3229-3240. CrossRef Medline 
Chiarandini DJ, Stefani E (1979) Electrophysiological identification of two types of fibres in rat extraocular muscles. J Physiol 290:453-465. Medline

Devoto SH, Melançon E, Eisen JS, Westerfield M (1996) Identification of separate slow and fast muscle precursor cells in vivo, prior to somite formation. Development 122:3371-3380. Medline

Drerup CM, Nechiporuk AV (2013) JNK-interacting protein 3 mediates the retrograde transport of activated c-Jun $\mathrm{N}$-terminal kinase and lysosomes. PLoS Genet 9:e1003303. CrossRef Medline

Engel AG, Ohno K, Sine SM (1999) Congenital myasthenic syndromes: recent advances. Arch Neurol 56:163-167. CrossRef Medline

Engel AG, Ohno K, Sine SM (2003) Sleuthing molecular targets for neurological diseases at the neuromuscular junction. Nat Rev Neurosci 4:339352. CrossRef Medline

Engel AG, Shen XM, Selcen D, Sine S (2012) New horizons for congenital myasthenic syndromes. Ann N Y Acad Sci 1275:54-62. CrossRef Medline

Epley KE, Urban JM, Ikenaga T, Ono F (2008) A modified acetylcholine receptor $\delta$-subunit enables a null mutant to survive beyond sexual maturation. J Neurosci 28:13223-13231. CrossRef Medline

Granato M, van Eeden FJ, Schach U, Trowe T, Brand M, Furutani-Seiki M, Haffter P, Hammerschmidt M, Heisenberg CP, Jiang YJ, Kane DA, Kelsh RN, Mullins MC, Odenthal J, Nüsslein-Volhard C (1996) Genes controlling and mediating locomotion behavior of the zebrafish embryo and larva. Development 123:399-413. Medline

Harel M, Kasher R, Nicolas A, Guss JM, Balass M, Fridkin M, Smit AB, Brejc K, Sixma TK, Katchalski-Katzir E, Sussman JL, Fuchs S (2001) The binding site of acetylcholine receptor as visualized in the X-ray structure of a complex between alpha-bungarotoxin and a mimotope peptide. Neuron 32:265-275. CrossRef Medline

Huzé C, Bauché S, Richard P, Chevessier F, Goillot E, Gaudon K, Ben Ammar A, Chaboud A, Grosjean I, Lecuyer HA, Bernard V, Rouche A, Alexandri N, Kuntzer T, Fardeau M, Fournier E, Brancaccio A, Rüegg MA, Koenig J, Eymard B, et al. (2009) Identification of an agrin mutation that causes congenital myasthenia and affects synapse function. Am J Hum Genet 85:155-167. CrossRef Medline

Ikenaga T, Urban JM, Gebhart N, Hatta K, Kawakami K, Ono F (2011) Formation of the spinal network in zebrafish determined by domainspecific pax genes. J Comp Neurol 519:1562-1579. CrossRef Medline

Jin TE, Wernig A, Witzemann V (2008) Changes in acetylcholine receptor function induce shifts in muscle fiber type composition. FEBS J 275:20422054. CrossRef Medline

Lefebvre JL, Jing L, Becaficco S, Franzini-Armstrong C, Granato M (2007) Differential requirement for MuSK and dystroglycan in generating patterns of neuromuscular innervation. Proc Natl Acad Sci U S A 104:24832488. CrossRef Medline

Luna VM, Brehm P (2006) An electrically coupled network of skeletal muscle in zebrafish distributes synaptic current. J Gen Physiol 128:89-102. CrossRef Medline

Luna VM, Wang M, Ono F, Gleason MR, Dallman JE, Mandel G, Brehm P (2004) Persistent electrical coupling and locomotory dysfunction in the zebrafish mutant shocked. J Neurophysiol 92:2003-2009. CrossRef Medline

Meyer A, Van de Peer Y (2005) From 2R to 3R: evidence for a fish-specific genome duplication (FSGD). Bioessays 27:937-945. CrossRef Medline

Milone M, Wang HL, Ohno K, Prince R, Fukudome T, Shen XM, Brengman JM, Griggs RC, Sine SM, Engel AG (1998) Mode switching kinetics produced by a naturally occurring mutation in the cytoplasmic loop of the human acetylcholine receptor epsilon subunit. Neuron 20:575-588. CrossRef Medline

Mishina M, Takai T, Imoto K, Noda M, Takahashi T, Numa S, Methfessel C, Sakmann B (1986) Molecular distinction between fetal and adult forms of muscle acetylcholine receptor. Nature 321:406-411. CrossRef Medline

Mongeon R, Gleason MR, Masino MA, Fetcho JR, Mandel G, Brehm P, Dallman JE (2008) Synaptic homeostasis in a zebrafish glial glycine transporter mutant. J Neurophysiol 100:1716-1723. CrossRef Medline

Mongeon R, Walogorsky M, Urban J, Mandel G, Ono F, Brehm P (2011) An acetylcholine receptor lacking both and subunits mediates transmission in zebrafish slow muscle synapses. J Gen Physiol 138:353-366. CrossRef Medline

Naganawa Y, Hirata H (2011) Developmental transition of touch response from slow muscle-mediated coilings to fast muscle-mediated burst swimming in zebrafish. Dev Biol 355:194-204. CrossRef Medline

Nashmi R, Dickinson ME, McKinney S, Jareb M, Labarca C, Fraser SE, Lester HA (2003) Assembly of alpha4beta2 nicotinic acetylcholine receptors assessed with functional fluorescently labeled subunits: effects of localization, trafficking, and nicotine-induced upregulation in clonal mammalian cells and in cultured midbrain neurons. J Neurosci 23:11554-11567. Medline

Ohno K, Wang HL, Milone M, Bren N, Brengman JM, Nakano S, Quiram P, Pruitt JN, Sine SM, Engel AG (1996) Congenital myasthenic syndrome caused by decreased agonist binding affinity due to a mutation in the acetylcholine receptor epsilon subunit. Neuron 17:157-170. CrossRef Medline

Ohno K, Tsujino A, Brengman JM, Harper CM, Bajzer Z, Udd B, Beyring R, Robb S, Kirkham FJ, Engel AG (2001) Choline acetyltransferase mutations cause myasthenic syndrome associated with episodic apnea in humans. Proc Natl Acad Sci U S A 98:2017-2022. CrossRef Medline

Ohno K, Engel AG, Shen XM, Selcen D, Brengman J, Harper CM, Tsujino A, Milone M (2002) Rapsyn mutations in humans cause endplate acetylcholine-receptor deficiency and myasthenic syndrome. Am J Hum Genet 70:875-885. CrossRef Medline

Ono F, Higashijima S, Shcherbatko A, Fetcho JR, Brehm P (2001) Paralytic zebrafish lacking acetylcholine receptors fail to localize rapsyn clusters to the synapse. J Neurosci 21:5439-5448. Medline

Ono F, Mandel G, Brehm P (2004) Acetylcholine receptors direct rapsyn clusters to the neuromuscular synapse in zebrafish. J Neurosci 24:54755481. CrossRef Medline

Papke RL, Ono F, Stokes C, Urban JM, Boyd RT (2012) The nicotinic acetylcholine receptors of zebrafish and an evaluation of pharmacological tools used for their study. Biochem Pharmacol 84:352-365. CrossRef Medline

Park JY, Ikeda H, Ikenaga T, Ono F (2012) Acetylcholine receptors enable the transport of rapsyn from the Golgi complex to the plasma membrane. J Neurosci 32:7356-7363. CrossRef Medline

Robinson KG, Viereck MJ, Margiotta MV, Gripp KW, Abdul-Rahman OA, Akins RE (2013) Neuromotor synapses in Escobar syndrome. Am J Med Genet A 161:3042-3048. CrossRef Medline

Tzartos SJ, Rand DE, Einarson BL, Lindstrom JM (1981) Mapping of surface structures of electrophorus acetylcholine receptor using monoclonal antibodies. J Biol Chem 256:8635-8645. Medline

van Raamsdonk W, van't Veer L, Veeken K, Heyting C, Pool CW (1982) Differentiation of muscle fiber types in the teleost Brachydanio rerio, the zebrafish: posthatching development. Anat Embryol 164:51-62. CrossRef Medline

Walogorsky M, Mongeon R, Wen H, Mandel G, Brehm P (2012a) Acetylcholine receptor gating in a zebrafish model for slow-channel syndrome. J Neurosci 32:7941-7948. CrossRef Medline

Walogorsky M, Mongeon R, Wen H, Nelson NR, Urban JM, Ono F, Mandel G, Brehm P (2012b) Zebrafish model for congenital myasthenic syndrome reveals mechanisms causal to developmental recovery. Proc Nat Acad Sci U S A 109:17711-17716. CrossRef Medline

Wang HL, Ohno K, Milone M, Brengman JM, Evoli A, Batocchi AP, Middleton LT, Christodoulou K, Engel AG, Sine SM (2000) Fundamental gating mechanism of nicotinic receptor channel revealed by mutation causing a congenital myasthenic syndrome. J Gen Physiol 116:449-462. CrossRef Medline

Weiss A, Schiaffino S, Leinwand LA (1999) Comparative sequence analysis of the complete human sarcomeric myosin heavy chain family: implications for functional diversity. J Mol Biol 290:61-75. CrossRef Medline

Wen H, Linhoff MW, Hubbard JM, Nelson NR, Stensland D, Dallman J, Mandel G, Brehm P (2013) Zebrafish calls for reinterpretation for the roles of $\mathrm{P} / \mathrm{Q}$ calcium channels in neuromuscular transmission. J Neurosci 33:7384-7392. CrossRef Medline

Won YJ, Ono F, Ikeda SR (2012) Characterization of $\mathrm{Na}+$ and $\mathrm{Ca}^{2+}$ channels in zebrafish dorsal root ganglion neurons. PLoS One 7:e42602. CrossRef Medline

Zimmermann L, May PJ, Pastor AM, Streicher J, Blumer R (2011) Evidence that the extraocular motor nuclei innervate monkey palisade endings. Neurosci Lett 489:89-93. CrossRef Medline 